\title{
Eritema nodoso
}

\section{Da consulta do Médico de Família ao diagnóstico}

\author{
CARLA PONTE,* FILIPA ALMADA LOBO*
}

\section{RESUMO}

Enquadramento: 0 Médico de Família, sendo muitas vezes o primeiro contacto dos utentes com o sistema de saúde, tem o papel de abordar inicialmente várias patologias e a necessidade de articular com outros niveis de cuidados. 0 eritema nodoso (EN) é uma síndroma clínica de etiologia múltipla. A atitude terapêutica requer a investigação das causas mais frequentes e do seu tratamento específico.

É a forma de paniculite mais comum, sendo importante 0 seu reconhecimento e estudo pelo médico de família.

Descrição do Caso: Mulher de 19 anos de idade, desempregada, Graffar Ve Apgar familiar 9. Recorreu ao Médico de Família por aparecimento de lesões nodulares, vinosas e dolorosas nos membros inferiores associadas a artralgias com cinco dias de evolução. Este constatou tratar-se de EN, tendo iniciado investigação e medicado a doente com um anti-inflamatório. Pela persistência e agravamento dos sintomas a doente foi ao Serviço de Urgência (SU) hospitalar, tendo sido submetida a uma radiografia do tórax e medicada com antibioterapia. Devido à manutenção do quadro clínico, recorreu novamente ao Médico de Família, que a referenciou ao hospital, onde ficou internada. Após o estudo analítico e imagiológico chegou-se ao diagnóstico de sarcoidose, tendo tido como primeira manifestação clínica o EN.

Discussão: 0 caso clínico apresentado pretende mostrar a articulação entre os cuidados primários e secundários de saúde, permitindo um estudo abrangente do EN.

Tem a particularidade de se rever as atitudes de abordagem médica, desde a avaliação clinica inicial até à escolha dos exames complementares de diagnóstico direccionado na investigação etiológica do EN.

Palavras chave: Eritema nodoso; Sarcoidose; Paniculite.

\section{ABSTRACT}

Context: The role of the family doctor, often the first contact that patients have with the health system, is to approach initially several pathologies and to articulate them with other health care levels.

Erythema nodosum (EN) is a multiple etiology clinical syndrome. The therapy requires a research on the most frequent causes and their specific treatment. It is the most frequent panniculitis version and, therefore, both its recognition and study by the family doctor are very important.

Case description: Nineteen years old woman, unemployed, with Graffar V and family Apgar 9. The woman visited her family doctor due to the appearance of painful, vinosa and nodular lesions in inferior limbs associated with artralgies with 5 days of evolution. The family doctor identified it as EN, initiated the investigation and medicated the patient with an anti-inflammatory. Patient symptoms persisted and worsened and, therefore, the patient went to the hospital emergency services, where she took a torax $x$-ray and was medicated with antibiotherapy.

Since the clinical condition kept persisting, the family doctor came once more at play, recommending her to go to the hospital, where she was interned.

After the analytical and imagiological studies, sarcoidosis was diagnosed, having as first clinical manifestation, the erythema nodosum.

Discussion: This clinical case aims to show the articulation between primary health care and secondary health care, allowing a broad study of Erythema nodosum. It gives the opportunity to review the medical approach, from the initial clinical investigation to the diagnosis exams that are important for EN etiologic research.

Keywords: Erythema nodosum Sarcoidosis; Panniculitis.

\section{INTRODUÇ̃̃O}

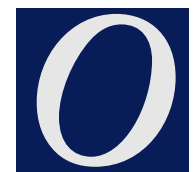

Médico de Família encontra-se diariamente perante dois aspectos inerentes à sua prática clinica: a abordagem inicial e sistemática das patologias e a articulação entre os diferentes níveis de cuidados.

*Interna do Internato de Medicina Geral e Familiar do Centro de Saúde da Senhora da Hora e do Centro de Saúde Horizonte, ULS Matosinhos
Este caso clínico pretende realçar a importância desses dois pontos na prestação de cuidados e na sua relevância para a continuidade dos mesmos. Uma das características do Médico de Família é a continuidade de cuidados prestados, o que torna fundamental a boa gestão de recursos e uma eficaz comunicação entre os diferentes niveis de cuidados. ${ }^{1}$

A Rede de Referenciação Hospitalar pretende regulamentar as relações entre as instituições de saúde, de modo a garantir o acesso a todos os doentes. É caracterizada essen- cialmente por três pilares: ${ }^{2}$

- Articulação em rede: pressupõe intercomunicação entre os diferentes niveis de cuidados.

- Complementaridade: o que permite aproveitar sinergias.

- Concentração de recursos: de modo a incrementar a rentabilidade desses recursos, com vista à melhoria da qualidade dos cuidados.

O Médico de Família deve funcionar como coordenador dos serviços prestados ao doente, devendo ser capaz de, perante cada situação, realizar uma abordagem inicial e de 
fazer a triagem dos casos que necessitam de intervenção de outros níveis de cuidados.

\section{DESCRICÃO DO CASO}

\section{Identificação}

RPAC, do sexo feminino, com 19 anos de idade, de raça caucasiana; vive em Custóias em união de facto, tem o $6^{\circ}$ ano de escolaridade e encontra-se desempregada.

\section{Antecedentes Pessoais}

Fisiológicos: Menarca aos 13 anos, apresentando desde então ciclos regulares; encontra-se a tomar anticonceptivos orais; teve uma gestação e um parto eutócico aos 17 anos.

Patológicos/Outros: Irrelevantes, sem hábitos medicamentosos (além da contracepção hormonal).

\section{Antecedentes Familiares}

Como antecedentes familiares relevantes apresenta: a mãe com epilepsia, a avó materna com diabetes tipo 2 e uma irmã com patologia cardíaca.

Pertence a uma família alargada, altamente funcional (Apgar familiar

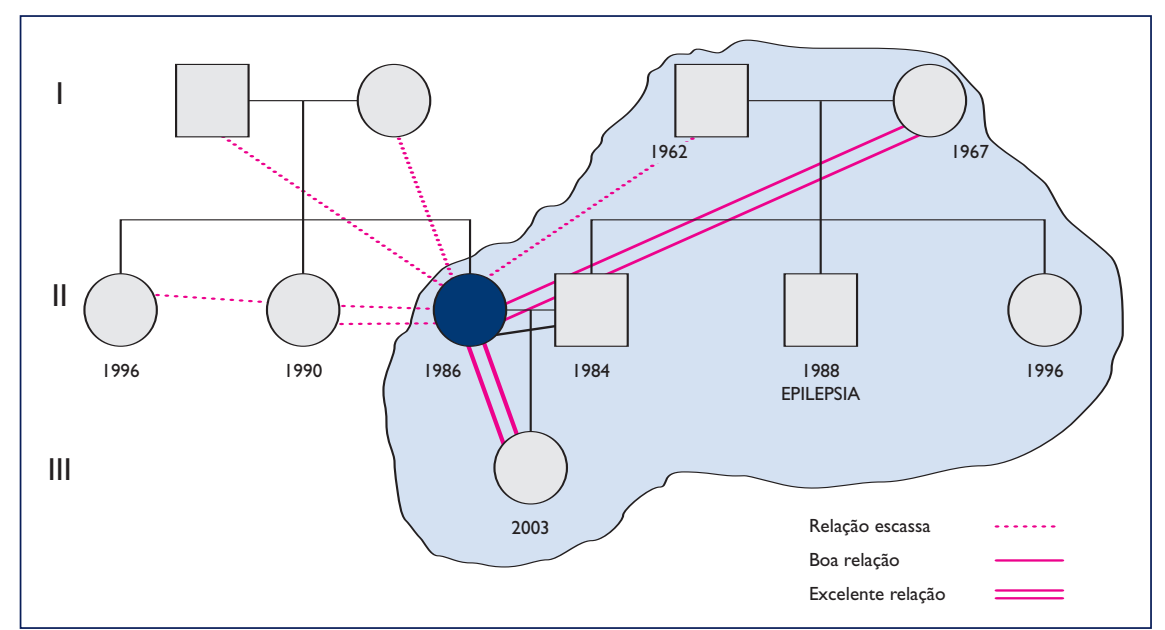

Figura 1. Genograma de 9), da classe V de Graffar (Figura 1$)$.

Através da análise do genograma, pode-se verificar que a doente vive actualmente com o companheiro e a sua família. Os laços mais fortes foram estabelecidos com a sogra e com a sua filha, apresentando afectividade muito escassa relativamente aos pais e irmãos.

\section{Problemas Sociais identificados:}

Mãe: alcoolismo crónico, maus-tratos aos filhos.

Pai: toxicodependência, tendo abandonado o lar enquanto a doente ainda era criança.

Irmã menor vive sozinha.

\section{$1^{\text {a }}$ Consulta (12/05/05)}

A doente recorre a esta consulta devido ao aparecimento de lesões eritematosas e dolorosas na face anterior das pernas, com cerca de cinco dias de evolução, e sem outras queixas. Ao exame objectivo encontra-se corada, hidratada e apirética. A auscultação cardíaca e pulmonar não revela qualquer alteração. As lesões que apresenta na face anterior das pernas são nodulares e eritematovioláceas. No restante exame físico 
(75,8\%) e uma linfopenia (13,3\%). Perante o atingimento do estado geral e manutenção do quadro o Médico de Família referenciou a doente para o SU hospitalar, com pedido de informação de retorno. A carta de referenciação foi devidamente redigida, com indicação dos antecedentes pessoais, hábitos medicamentosos e relato da história actual e das intervenções clínicas realizadas até esse momento.

Dessa referenciação resulta o internamento da doente, descrito abaixo sucintamente (a descrição é baseada na nota de alta e na consulta do processo clínico hospitalar):

\section{Internamento $(20 / 05 / 05$ a 08/06/05)}

Do estudo efectuado foram detectadas as seguintes alterações:

- Anemia Normocítica Normocrómica ligeira $(11,2 \mathrm{~g} / \mathrm{dl})$, VS aumentada $(102 \mathrm{~mm} / \mathrm{h})$.

- Rx e TAC pulmonares: adenopatias peri-hilares.

- Razão $\mathrm{CD}_{4} / \mathrm{CD}_{8}=7,33$ no lavado bronco-alveolar.

\section{Diagnóstico:}

- Sarcoidose - apresentação por EN.

\section{Tratamento Instituído:}

Prednisolona 30 mg/dia

\section{Alta:}

A doente teve alta, com melhoria clínica e regressão das lesões do EN, mantendo em ambulatório a corticoterapia. Foi programada consulta de Medicina Interna para 07/07/05.

Cerca de 1 mês após alta hospitalar, a doente foi à consulta programada de Medicina Interna, não sendo, nesta data, evidentes as lesões de EN, nem se apresentando analiticamente, com anemia. Foi decidido, então, proceder à redução progres- siva da corticoterapia e à repetição da TAC torácica.

Foi encaminhada, com nota de alta, para o Médico de Família, para vigilância e acompanhamento da evolução clínica.

\section{DISCUSSÃo}

Este caso clínico permite-nos reflectir sobre os dois pontos inicialmente referidos: a abordagem inicial do EN e a articulação de cuidados de saúde.

\section{Abordagem Inicial do Eritema Nodoso}

Na primeira abordagem o Médico de Família fez uma análise da situação, tendo colocado, como hipótese diagnóstica mais provável, o EN, iniciando a investigação para esta patologia. Há a referir que essa abordagem inicial deve incluir:

- Colheita de história clínica com informação sobre doenças recentes/problemas de saúde (infecções do tracto respiratório superior; infecções estreptocócicas, etc.), medicação instituída ou em curso, sintomas associados (artralgias, diarreia, sintomas respiratórios) e sua relação temporal com o aparecimento das lesões;

- Exame físico cuidadoso, com reconhecimento das lesões nodulares típicas - na maioria das vezes o diagnóstico é clínico dispensando a realização de biópsia que confirmaria a existência de uma paniculite septal; - Hemograma, velocidade de sedimentação;

- Título Anti-estreptolisina O (duas colheitas com intervalo de duas a quatro semanas);

- Prova Tuberculina;

- Rx Pulmonar: tem um papel particular na exclusão de processos infecciosos como a tuberculose e a sar- coidose, que decorre com adenopatia hilar bilateral em $50 \%$ dos casos. ${ }^{3}$

- Com esta investigação inicial pretende-se procurar a etiologia do EN. ${ }^{4-6} \mathrm{O}$ EN é uma paniculite que afecta o tecido celular subcutâneo, com inflamação dos vasos sanguíneos septais o que se traduz por sinais de uma vasculite (nódulos eritematosos, tensos e dolorosos com localização preferencial nas faces extensoras das pernas). Este é habitualmente idiopático, no entanto pode ser o primeiro sinal de uma doença sistémica, como a tuberculose, a sarcoidose, a doença inflamatória intestinal ou o cancro; certas drogas como os anticonceptivos orais e alguns antibióticos podem também ser a causa do EN (Quadro I). ${ }^{7}$

Diversos estudos têm apontado para uma etiologia idiopática em cerca de $55 \%$ dos casos de EN. ${ }^{8}$ No entanto, é necessário reconhecer as principais causas de EN secundário. ${ }^{8.9} \mathrm{Na}$ Europa ainda são as infecções estreptocócicas a principal causa (8 a 63\%). A sarcoidose é também

\section{QUADRO I}

\begin{tabular}{|ll|}
\hline \multicolumn{2}{|c|}{ CAUSAS DE ERITEMA NODOSO } \\
\hline Infecções & Infecções estreptocócicas \\
bacterianas & Tuberculose \\
& Chlamydia \\
& Salmonela \\
\hline Infecções víricas & Mononucleose \\
& Hepatite B \\
& HIV \\
& Hepatite C \\
\hline Drogas & Sulfonamidas \\
& Sulfonilureias \\
& Anticonceptivos orais \\
& Penicilinas \\
\hline Doenças & Sarcoidose \\
sistémicas & Lúpus Eritematoso Sistémico \\
& Enteropatias (Colite ulcerosa; \\
& Doença de Crohn) \\
\hline
\end{tabular}


prevalente (11 a 33\%), assim como a doença de Crohn e colite ulcerosa. Nestes últimas duas etiologias há geralmente associação do EN com diarreia. ${ }^{8.9}$

É de realçar que em Portugal a hipótese de tuberculose é sempre de colocar $^{7,10}$ - nos estudos consultados a prevalência de tuberculose é de $0,5 \%$ a $18 \%$. Em Portugal não existem estudos sobre a prevalência de EN por etiologia, mas uma vez que se conhece a realidade em termos de prevalência de tuberculose, que é elevada, esta etiologia deve ser sempre descartada.

Neste caso, deveria ter sido pedido o Rx pulmonar e a Prova de Mantoux, numa primeira abordagem, com o objectivo de se descartar as hipóteses de tuberculose e de sarcoidose.

O diagnóstico obtido foi sarcoidose, baseado nos seguintes critérios: EN, linfadenopatia hilar bilateral e exames analíticos compativeis (velocidade de sedimentação e proteína $\mathrm{C}$ reactiva aumentadas). Não foi efectuada a confirmação histológica, mas obteve-se no lavado bronco-alveolar uma alveolite linfocítica com uma razão $\mathrm{CD}_{4} / \mathrm{CD}_{8}=7,33$.

O diagnóstico de sarcoidose é baseado nos seguintes critérios: 1) clínica compatível com alterações radiológicas; 2) evidência histológica de granulomas não caseosos; 3) exclusão de outras doenças capazes de produzir alterações histológicas semelhantes. No caso de não ser possivel obter dados histológicos, os dados do lavado bronco-alveolar e o valor da Enzima Conversora da Angiotensina (ECA) podem fornecer informação diagnóstica adicional..$^{11-13}$

$\mathrm{O}$ valor da relação $\mathrm{CD} 4 / \mathrm{CD} 8$ superior a 3,5 no lavado bronco-alveolar pode confirmar o diagnóstico e obviar a necessidade de confirmação com biópsia. Este dado tem uma sensibilidade de $52-59 \%$ e especificidade de 94 a $96 \% . .^{11-13}$

\section{Articulação de cuidados}

Neste caso clínico está descrito o percurso da doente desde o início do quadro clínico até ao diagnóstico e terapêutica. O Médico de Família foi, como na maioria dos casos, o primeiro contacto da doente com o sistema de saúde. Passados alguns dias a doente recorre, por sua iniciativa, ao SU hospitalar, por agravamento da sintomatologia. A nível hospitalar foi realizado Rx pulmonar e instituída antibioterapia. Faz-se notar que não foi enviada qualquer informação de retorno, o que impossibilitou o Médico de Família de conhecer o resultado do $\mathrm{Rx}$ efectuado e o raciocínio clínico levado a cabo para a instituição da terapêutica.

Com a evolução da doença, a manutenção dos sintomas e o atingimento do estado geral, a doente recorre novamente ao seu Médico de Família. Nesta segunda avaliação o médico tem alguns resultados analíticos e novos dados do exame objectivo, que em conjunto levam à decisão de referenciação hospitalar. Os motivos desta referenciação foram o atingimento do estado geral (traduzido-se por prostração e astenia), alertando-a para a presença de uma doença sistémica, juntamente com conhecimento de que o EN podia ser uma forma de expressão de várias patologias sistémicas. Perante este quadro a referenciação para o nível de cuidados secundário possibilitaria um diagnóstico mais atempado. Nesta referenciação foi crucial o papel da carta de referenciação pormenorizada, com solicitação de informação de retorno.

A carta de referenciação é um canal de comunicação que o Médico de Família deve aprimorar, pois muitas vezes dela depende a resposta dos cuidados de saúde secundários. É essencial fornecer todos os dados relacionados com o doente referenciado, de modo contribuir para uma abordagem global por parte do médico hospitalar.

A Figura 2 pretende ilustrar a abordagem inicial e seguimento deste caso clínico, tendo por base a estrutura de articulação de cuidados já referida. A destacar que por vezes a complexidade das patologias e sua múltipla etiologia provável pode levar a uma deficiente investigação inicial, como aconteceu, nesta caso, com a ausência do pedido do Rx de tórax. Foi lembrada a ausência de informação de retorno após a ida ao SU, relembrando a sua utilidade no seguimento e avaliação diagnóstica de qualquer caso clínico.

A articulação de cuidados não termina com este episódio de doença, deve manter-se durante o acompanhamento da doente, com comunicação entre a consulta hospitalar e consulta do Médico de Família; esta comunicação bidireccional pode ser facilitada com a informatização do processo clínico, evoluindo progressivamente para uma articulação que se pretende ideal.

\section{CONCLUSÃO}

Com a descrição deste caso clínico pretende-se destacar que a actuação inicial do Médico de Família é de extrema importância para o desfecho da situação clínica. Sem dúvida a abordagem inicial de qualquer patologia deve ser cuidadosa e ponderada com base nos dados clínicos, diagnósticos prováveis e conhecimento sobre a patologia e suas etiologias.

Por outro lado, a relação de empatia entre o médico e o doente assu- 


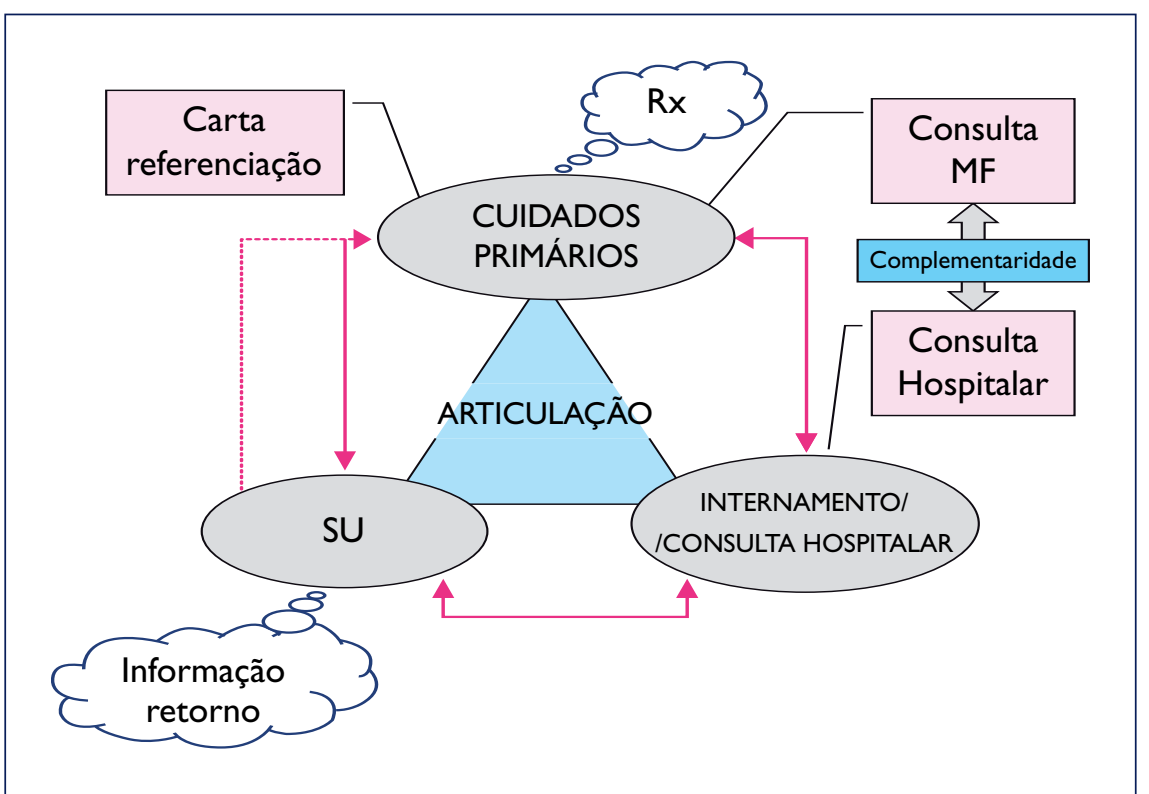

Figura 2. Articulação entre os Cuidados Primários e Secundários

me um papel de destaque no seguimento do doente, garantindo a continuidade de cuidados, com integração de todas as informações essenciais para tomar decisões, como a da referenciação.

A articulação entre os cuidados primários e secundários de saúde foi também ilustrada neste caso clínico, realçando a importância da carta de referenciação e da informação de retorno, que garantem um pressuposto essencial para o funcionamento desta articulação: a comunicação.

\section{REFERÊNCIAS BIBLIOGRÁFICAS}

1. Sá A. Filosofia e prática da clínica geral: consequências da sua especificidade. Rev Port Clin Geral 1995;12: 47-53.

2. Direcção Geral da Saúde. Direcção dos Serviços de Planeamento. A rede de referenciação hospitalar. Lisboa: DGS; 2001. Disponivel em: URL: http://www.dgs.pt/ upload/membro.id/ficheiros/i005661.pdf [acedido em 9/10/2007].

3. Garcia-Porrúa C, González-Gay MA,
Vázquez-Caruncho M, López-Lazaro L, Lueiro M, Fernández M, et al. Erythema nodosum: etiologic and predictive factors in a defined population. Arthritis Rheum 2000 Mar; 43 (3): 584-92.

4. Psychos DN, Voulgari PV, Skopouli FN, Drosos AA, Moutsopoulos HM. Erythema nodosum: the underlying conditions. Clin Rheumatol 2000; 19 (3): 212-6.

5. Brodell R, Mehrabi D. Underlying causes of erythema nodosum: lesions may provide clue to systemic disease. Postgrad Med 2002; 108 (6): 147-9.

6. Requena L, Requena C. Erythema nodosum. Dermatol Online J; 8 (1): 4.

7. Shwartz RA, Nervi S. Erythema nodosum: a sign of systemic disease. Am Fam Physician 2007 Mar 1; 75 (5): 695-700.

8. Cribier B, Caille A, Heid E, Grosshans E. Erythema nodosum and associated diseases: a study of 129 cases. Int J Dermatol 1998 Sep; 37 (9): 667-72.

9. Mert A, Ozaras R, Tabak F, Pekmezci S, Demirkesen C, Ozturk R. Erythema nodosum: an experience of 10 years. Scand J Infect Dis 2004; 36 (6-7): 424-7.

10. Mert A, Ozaras R, Tabak F, Ozturk R. Primary tuberculosis cases presenting with erythema nodosum. J Dermatol 2004 Jan; 31 (1): 66-8.

11. Statement on sarcoidosis. Joint Statement of the American Thoracic Society
(ATS), the European Respiratory Society (ERS) and the World Association of Sarcoidosis and Other Granulomatous Disorders (WASOG) adopted by the ATS Board of Directors and by the ERS Executive Committee, February 1999. Am J Respir Crit Care Med 1999 Aug; 160 (2): 736-55.

12. Costabel U. Sarcoidosis: clinical update. Eur Respir J Suppl 2001 Sep; 32: 56s-68s.

13. Wu JJ, Schiff KR .Sarcoidosis. Am Fam Physician 2004 Jul 15; 70 (2): 312-22.

\section{Endereço para correspondência}

Carla Mónica Faria Ponte

Travessa Avilhó, no 373

4460-689 Custóias - Matosinhos

Telf: 229543549

Tlm: 916079495

E-mail: carlaponte77@hotmail.com

Recebido em: 07/09/06

Aceite para publicação em: 02/10/07 